\title{
Ultrasensitive detections in atomic and molecular physics: demonstration in molecular overtone spectroscopy
}

\author{
Jun Ye,* Long-Sheng Ma, ${ }^{\dagger}$ and John L. Hall ${ }^{\ddagger}$ \\ JILA, University of Colorado and National Institute of Standards and Technology, Boulder, Colorado 80309-0440
}

Received May 28, 1997; revised manuscript received August 13, 1997

\begin{abstract}
We consider several highly sensitive techniques commonly used in detection of atomic and molecular absorptions. Their basic operating principles and corresponding performances are summarized and compared. We then present our latest results on the ultrasensitive detection of molecular overtone transitions to illustrate the principle and application of the cavity-enhanced frequency-modulation (FM) spectroscopy. An external cavity is used to enhance the molecular response to the light field, and an FM technique is applied for shotnoise-limited signal recovery. A perfect match between the FM sideband frequency and the cavity free spectral range makes the detection process insensitive to the laser-frequency noise relative to the cavity, and, at the same time, overcomes the cavity bandwidth limit. Working with a 1.064- $\mu \mathrm{m} \mathrm{Nd:YAG} \mathrm{laser,} \mathrm{we} \mathrm{obtained}$ sub-Doppler overtone resonances of $\mathrm{C}_{2} \mathrm{HD}, \mathrm{C}_{2} \mathrm{H}_{2}$, and $\mathrm{CO}_{2}$ molecules. A detection sensitivity of $5 \times 10^{-13}$ of integrated absorption $\left(1 \times 10^{-14} / \mathrm{cm}\right)$ over 1-s averaging time has been achieved. (C) 1998 Optical Society of America [S0740-3224(97)02012-2]
\end{abstract}

OCIS codes: $300.6390,020.0020$.

\section{INTRODUCTION}

Ultrasensitive detection of optical absorption by atomic/ molecular samples can be approached from two directions: either the intrinsic absorption signal (contrast) is enhanced or the background noise is reduced, ideally to the fundamental quantum limit. An optical cavity placed around the absorber is an effective technique to enhance the absorption contrast, as is conveniently explained in terms of extending the effective cell length. From another viewpoint, for a given noise generated by transmitted dc light on the detector, the high internal buildup field of the cavity elicits a strong molecular radiation, which is resonantly coupled out for detection.

The ultimate detection sensitivity is achieved when we are able to observe each absorption event, with the noise basically limited by the uncertainty of event occurrence, namely, the shot noise in the signal. To avoid excessive low-frequency noise of technical origins, signal detection should employ ac methods, comparing on-resonant and off-resonant cases in quick succession. By simultaneously obtaining and subtracting these cases, one provides a signal channel with no output unless there is a resonance. This is conveniently accomplished by use of the frequency-modulation (FM) technique.

To tailor the FM method for use in the presence of an enhancement cavity, one arranges the FM sideband offset frequency to match the cavity free-spectral-range splitting frequency so that the optical heterodyne sidebands and carrier are transmitted through the cavity via adjacent axial orders. A remarkable property of this configuration is the suppression of any detection of laserfrequency noise with the transmitted light. This enables profitable use of cavity finesse in the range above 10000 , without a noise penalty.

Using this detection scheme, we have now achieved a noise-equivalent absorption sensitivity of $5 \times 10^{-13}$ (1 $\left.\times 10^{-14} / \mathrm{cm}\right)$ at $1-\mathrm{s}$ averaging time. This sensitivity is demonstrated in the high signal-to-noise ratio $(\mathrm{S} / \mathrm{N})$ recovery of sub-Doppler resonances of weak molecular overtone $\left(\mathrm{C}_{2} \mathrm{H}_{2}, \mathrm{C}_{2} \mathrm{HD}\right.$, and $\left.\mathrm{CO}_{2}\right)$ transitions at $1.064 \mu \mathrm{m}$. A high sensitivity is desired for studying "forbidden" transitions or for detecting substances with very small absorption, owing to either low concentration or weak intensity. Applications of sensitive detection cover many different fields such as fundamental physics, atmospheric optics, trace-gas monitoring, molecular dynamics, reaction kinetics, and biology. In our application we seek a high sensitivity to use in developing visible-frequency standards based on a rich spectrum of attractive and yet extremely weak molecular overtone transitions.

The paper is organized in the following structure. First, we compare various sensitive detection techniques and their corresponding sensitivity limits. While methods of high-resolution spectroscopy are highly valuable in their own right, here we focus on the sensitivity aspects of various experimental approaches, in comparison with our method. We should bear in mind that each detection technique has its unique advantages and so may be the most effective method for one particular application. We then briefly present in Section 3 the detection principle of the cavity-enhanced FM technique. Experimental results are shown in Section 4. Finally in Section 5 we conclude with a summary and discussion of the technique and an outlook for its future applications.

\section{COMPARISON AMONG VARIOUS SENSITIVE DETECTION METHODS}

\section{A. Direct Absorption (Homodyne) Measurement}

Beer's absorption law states that light passing through an atomic/molecular sample of length $L$ will be attenuated 
by the factor $\exp (-\alpha L)$. The physical origin of this attenuation process is the destructive interference between the incident radiation and the electric field generated by the coherently driven sample dipole moments. ${ }^{1}$ Therefore the direct absorption is an intrinsically microscopic (quantum-mechanical) effect. The name, homodyne detection, arises from the fact that the two interfering fields have the same frequency. The maximum sensitivity condition will arise if the probe field has no amplitude noise beyond its intrinsic quantum fluctuation. Given a detection bandwidth of $B$, the minimum detectable absorption signal under this shot-noise limit is

$$
(\alpha L)_{\min }=\left(\frac{2 e B}{\eta P_{0}}\right)^{1 / 2},
$$

where $e$ is the electron charge, $\eta$ is the photodetector responsivity in amperes per watt, and $P_{0}$ is the incident radiation power. This sensitivity can be as high as 1 $\times 10^{-8}$ at 1 -s averaging for $P_{0}=1 \mathrm{~mW}$ and a reasonable $\eta(\sim 0.8 \mathrm{~A} / \mathrm{W}$ at $1.064 \mu \mathrm{m})$. In practice, however, noises of various technical origins tend to become dominant in the low-frequency ranges. A noisy background severely affects the measurement of a small change of signal and so limits the detection sensitivity. It is quite usual to lose several orders of magnitude relative to the shot-noise limit. In order to reduce the low-frequency noises, one can use modulation techniques, either on the laser amplitude or frequency, to encode and detect the absorption at a higher frequency and with a narrower bandwidth. Zero-background detection techniques are also quite often used in avoiding excessive noises. Alternatively a differential detection technique that suppresses noises with dual beams is also attractive. Besides reducing noises, improvements on the detection sensitivity can be directly approached by enhancing the signal itself. This leads to considerations of increasing sample path length by multiple-pass cells and intracavity enhancement.

\section{B. Photothermal Spectroscopy}

Photothermal spectroscopy is simple, convenient, and widely applicable. ${ }^{2}$ The underlying principle is that the heat transferred from the absorbed optical power to the sample via various de-excitation channels can cause subsequent macroscopic changes. Such thermal effects can be manifested and measured, for example, in the forms of temperature rise (photothermal calorimetry), pressure change and/or volume expansion (photoacoustic effect), change of optical properties such as the refractive index (probe-beam refraction, diffraction, or deflection, and probe-beam homodyne interference), and thermalemission change (photothermal radiometry). This technique of photothermal spectroscopy has in principle a zero background and is generally more sensitive than the direct absorption measurement. However, the significant amount of energy associated with the macroscopic heat transfer limits the sensitivity of photothermal spectroscopy. In the case of photoacoustic detection, noises of technical origins such as fluctuations of laser intensity and pointing direction, undesired absorption, and vibration and acoustic disturbance still far exceed the fundamental noise limit set by the Brownian-motion noise and shot-noise (statistical fluctuations) nature of the excitation and deexcitation processes. These similar technical noises also limit attainable sensitivities of the thermallensing detection technique and the optical phase-shift measurement in a two-beam interferometer. The obtained sensitivities are generally of the order of $10^{-4}-10^{-9}$ fractional absorption $\left(10^{-5}-10^{-10} / \mathrm{cm}\right) .^{3}$ Similar sensitivity limits are also obtained with other macroscopic techniques such as the optogalvanic effect, the optical rotation effect (dichroic absorption), and the change of photoconductivity.

\section{Polarization Spectroscopy}

Polarization spectroscopy ${ }^{4}$ is in principle a zerobackground detection method used in nonlinear spectroscopy. Absorption-induced polarization changes of light can be measured at an extremely small level, significantly improving the detection sensitivity. However, any practical polarizer has a finite extinction ratio $(\varepsilon)$, which may limit the attainable sensitivity. In fact, if the shot noise is the dominant noise source in the detection process, then the polarization technique offers no advantage compared with the direct absorption measurement. (Of course, if the detection power is shot-noise limited and it is larger than what a conventional photodetector would normally accept, then it is advantageous to use polarization spectroscopy.) This is because for the polarization spectroscopy to recover a small absorption signal, it will also rely on the heterodyne (or homodyne) product between the probe beam and the local oscillator. Only when the optical power is increased to a certain level where the technical noise dominates can the polarization spectroscopy reduce the noise level by a factor of $\sqrt{\varepsilon}$, with $\varepsilon \approx 10^{-7}$ for a good polarizer. The sensitivity limit of polarization spectroscopy therefore follows Eq. (1).

\section{Fluorescence Detection}

Resonance fluorescence is a highly sensitive technique for detecting small numbers of atoms and molecules. ${ }^{5}$ It has also been applied in the spectroscopy of trapped single ions. $^{6,7}$ In analytical chemistry and biology, resonance fluorescence is used extensively for efficient detection and identification of single molecules. ${ }^{8}$ Fluorescence is also an efficient detection technique for multiphoton absorption. The sensitivity of the fluorescence-detection technique depends on several factors including the characteristics of the decay channels of the excited states, namely, that the fluorescence branching ratios are favorable and the quenching processes are not significant. For maximum detected signal, the decay photons must be collected over as large a solid angle as possible. A reasonably well-designed optical system can collect $\sim 10 \%$ of the radiated quanta after having filtered out stray scattered light. With quantum efficiencies in the range of $20 \%$ for the cathode surfaces of modern photomultipliers in the visible domain, one can expect a photoelectron event for every 50 or so fluorescent decays. The dark current of the quantum detector can be as small as a few counts per second for cooled photomultipliers with bialkali cathodes. Photon-counting techniques permit one to detect a light level some (suppose we say ten) counts per second above this background. If the incident cw laser power is $1 \mathrm{~mW}$ 
and $10 \%$ of the absorption events result in photons of the desired wavelength, an attenuation of this beam by $10^{-10}-10^{-11}$ can be detected. For multiphoton absorptions, this sensitivity can be further enhanced with the use of a higher optical power. However, various phenomena may degrade the $\mathrm{S} / \mathrm{N}$, particularly if the excitation and detection are at the same wavelength. Then the most severe limitation on the detection sensitivity is caused by the background spurious scattering light, which usually dominates stray ambient light, even with careful baffling and shielding. By discriminating the event photoelectrons from the background noise, photoncorrelation spectroscopy, such as photon-burst spectroscopy or the time-interval-distribution analysis, often help to enhance the effectiveness of the fluorescence detection. ${ }^{9}$

\section{E. (Non-) Resonant Multiphoton Ionization Spectroscopy}

This technique has been used very efficiently in the fields of surface analysis, isotope analysis, rare-element detection, and single atom or molecule detection. ${ }^{10}$ For an absorbing transition with an ionizable upper level, ionization spectroscopy can be the most sensitive detection technique simply because both the collection and detection efficiency for the charged particles could approach unity. This means that every photon-absorption event can be made detectable, by measurement of the total ion counts, mass spectroscopy of ions, or analysis of the kinetic energy of ions or electrons. The disadvantage lies in the requirement of a large photon flux so that the ionization rate of the excited state is sufficient, compared with other relaxation channels of its decay into lower levels.

\section{F. Balanced Beam/Differential Receiver}

With a dual-beam approach the information about the laser noise can be measured twice, and therefore it is possible to completely eliminate the technical noise and approach the fundamental limit of shot noise by use of clever designs of optoelectronic receivers. Conventional dual-beam detection systems use delicate optical balancing schemes, ${ }^{11}$ which are often limited by the noise and drift of beam intensities, residual interference fringes, drift in amplifiers, and spatial inhomogeneity in the detectors. This is the reason why present dual-beam optical balancing schemes often employ some sort of modulation strategies. Electronic autocancellation of the photodetector currents has provided near-shot-noiselimited performance. ${ }^{12}$ Indeed a noise-equivalent absorption of $4.2 \times 10^{-8}$ was achieved with $1.2 \mathrm{~mW}$ of optical power and $1-\mathrm{Hz}$ bandwidth, roughly $\sqrt{2}$ above the shot-noise limit. This technique is straightforward and requires no modulation, although it does not fully solve the problem of baseline drift.

\section{G. External-Cavity Spectroscopy: Comparison Between Cavity-Ring-Down and Direct-Cavity-Transmission Measurements}

Sensitivity enhancement of absorption measurements has been accomplished by use of long multipass absorption cells. ${ }^{13}$ Kastler suggested a Fabry-Perot cavity could be used because its transmission is sensitive to small variations of its inside absorption. ${ }^{14}$ The use of the externalcavity technique was later extended to the highresolution sub-Doppler spectroscopy. ${ }^{15-17}$ The advantages of using an external optical resonator are manifold. First, the light travels many times through the intracavity absorber so that the effective absorption length is increased by the factor of $(2 \times$ finesse $/ \pi)$. This directly increases the detection sensitivity. Second, the cavity builds up its intracavity power. This allows the use of low-power lasers for the input, even for the weak transitions that require large intensities for sub-Doppler high-resolution saturation spectroscopy. For a given signal, effectively the cavity reduces the output power level that needs to be handled by a photodetector. In addition, the geometrical self-cleaning and matching of the two counterpropagating waves inside the cavity are important both for eliminating pointing-direction-related noises and for obtaining narrow and unshifted resonance lines. ${ }^{18}$ In another role, a stable cavity helps to prestabilize the laser frequency when it is locked on a cavity resonance, thereby reducing the detection noise. For the purpose of establishing a stable and repeatable optical frequency reference, it is also attractive to separate the absorber from the laser to avoid various coupled instability problems associated with intra-laser-cavity absorption.

The shot-noise-limited sensitivity in Eq. (1) of direct absorption measurement can therefore be multiplied by a factor of $(2 \times$ finesse $/ \pi)$ with the use of an optical resonator. With a cavity finesse of 100000 , it is possible to reach a sensitivity in the $10^{-13}$ range, as long as one avoids various technical noises associated with the highfinesse cavity. The so-called cavity-ring-down spectroscopy ${ }^{19}$ takes advantage of the cavity memory to avoid laser technical noise. It measures the cavity fielddecay time (which is a function of the intracavity absorption) once the input laser field is switched off, hence avoiding the laser amplitude noise. Given an adequately fast digitizer to record the ring-down waveform with a sufficient resolution, it appears that the shot-noise limit can be approached. Unfortunately the on-resonance versus off-resonance decay-time measurements are separated in time, and it is their difference that contains the desired information. This time separation allows the influence of many unwelcome noise sources including geometry-dependent ring-down rates. The best reported sensitivity so far is $9 \times 10^{-8}\left(7 \times 10^{-10} / \mathrm{cm}\right){ }^{20}$

Another effective technique for avoiding the light noise at low frequency is to lock the laser frequency tightly on the corresponding cavity resonance and then modulate the cavity mode around the desired molecular resonance while monitoring the cavity transmission. Essentially this approach is a simple lock-in derivative-line-shape recovery process. In order for this method to be successful it is important to have a super-tight frequency lock loop between the laser and the cavity since any laserfrequency noise relative to the cavity will be converted to amplitude noise in detection. A piezoelectric transducer (PZT) mounted on one of the cavity mirrors can be used to modulate the cavity length, and the laser will track this modulation. The modulation frequency is usually limited to the audio range owing to the mechanical resonance 
and roll-off of the PZT and mirror assembly. Depending on the laser (amplitude) noise spectral distribution, the modulation frequency may be too low to reach the shotnoise-limited spectral region. However, attractive results have already been obtained in our experiment. Our solid-state Nd:YAG laser is locked onto our high-finesse (100 000) cavity with $\sim 1-\mathrm{mHz}$ relative linewidth. A cavity dither (at $500 \mathrm{~Hz}$ ) and lock-in detection yields a detection sensitivity of $3 \times 10^{-11}\left(6.4 \times 10^{-13} / \mathrm{cm}\right)$ at 1 -s averaging. This is already more than 1000 times better than the ring-down result.

\section{H. Intracavity Spectroscopy}

It was realized in the early days of laser development that a laser cavity can be used to greatly enhance the absorption detection sensitivity. ${ }^{21}$ Basically the advantage comes from the multipass effect and the delicate balance between the laser gain and intracavity absorption (threshold effect and the mode-competition effect). ${ }^{22} \mathrm{Al}$ though it is still a complicated system involving the dynamical evolution of laser modes, it is one of the most sensitive detection techniques for absorption. Operating with a single-lasing mode, or when the intracavity absorber has a linewidth wider than the entire laser bandwidth, intracavity absorptions can be monitored directly with the laser output power or indirectly with respect to a reference loss, to the laser emission spectrum, to the laser-magnetic resonance, to the lasing dynamics, etc. A reasonable enhancement is obtained only when the laser operation is near threshold where various instabilities tend to limit the sensitivity. When a narrower band absorber is used instead, the broadband laser gain is not much affected by the narrow line absorption. For such a multimode laser the resultant emission spectrum relates to the intracavity absorption with a high sensitivity, limited only by the degree of nonlinear mode coupling, the laser pulse duration, and more fundamentally, the broadband cavity loss and the quantum noise (the ratio of induced and spontaneous emission in the laser mode). For the best sensitivity, other unwanted frequencyselective losses should be avoided. To avoid spurious resonance features in the spectrum, all intracavity optics should be clean and free of interference. Laser intensity noise should be kept small. The highest sensitivity reported so far using the intracavity spectroscopy is 3 $\times 10^{-10} / \mathrm{cm}^{23}$ A major advantage of intracavity spectroscopy is that it covers simultaneously a broad spectral width, similar to the laser gain bandwidth.

\section{Frequency Modulation (Wavelength Modulation)}

FM spectroscopy is one of the most powerful spectroscopic techniques available for supersensitive and high-speed detections of weak absorption signals. In principle, FM spectroscopy offers a detection sensitivity approaching that of Eq. (1), limited only by the fundamental quantum noise. A side advantage of this technique is that linewidth broadenings associated with low-frequency modulation processes are not present. The widespread FM spectra allow each individual component to interact with the spectral features of interest and thereby preserve the ultrahigh resolution capability of contemporary narrow- linewidth lasers. The high bandwidth associated with the radio-frequency (rf) modulation enables rapid signal recovery.

Modulation techniques are of course often used to extract weak signals from a noisy background. Small resonance information is encoded into a frequency region where the technical noise is relatively weak. Various modulation schemes allow one to compare on-resonant and off-resonant cases in quick succession. Subsequent demodulations (lock-in detection) then simultaneously obtain and subtract these two cases, hence generating a signal channel with no output unless there is a resonance. Lorentzian signal recovery with the frequencymodulation method has been well documented. ${ }^{24}$ The associated lock-in detection can provide the first, second, and third derivative type of output signals. However, in this type of modulation spectroscopy the modulation frequency is often chosen to be relatively low to avoid distortions on the spectral profile by the auxiliary resonances associated with modulation-induced spectral sidebands. An equivalent statement is that the line is distorted because it cannot reach an equilibrium steady state in the face of the rapidly tuning excitation. This low-frequency operation (either intensity chopping or derivative lineshape recovery) usually is still partly contaminated by the technical noise and the achievable $\mathrm{S} / \mathrm{N}$ is thereby limited. To recover the optimum signal size, large modulation amplitudes (comparable to the resonance width) are also employed, leading to a broadened spectral linewidth. Therefore the intrinsic line shape is modified by this signal-recovery process and the direct experimental resolution is compromised.

A different modulation technique was then proposed and developed in the microwave magnetic resonance spectroscopy and similarly in the optical domain. ${ }^{25-27}$ The probing field is phase modulated at a frequency much larger than the resonance linewidth under study. Subsequent heterodyne and $\mathrm{rf}$ phase-sensitive detection yield the desired signal. The high sensitivity associated with the FM spectroscopy is mainly due to its high modulation frequency, usually chosen to lie in a spectral region where the amplitude noise level of the laser source approaches the quantum (shot-noise) limit. The redistribution of some of the carrier power to its FM sidebands causes only a slight penalty in the recovered signal size. In practice, some systematic effects exist to limit the ultimate FM sensitivity. Spurious noise sources can include residual amplitude noise at modulation frequency, ${ }^{28}$ laser excess noise, and étalon fringes in the optical system. ${ }^{29}$ A number of techniques have been developed to overcome these problems. The residual amplitude-modulation (AM) problem can be actively servo controlled down to the shotnoise limit. ${ }^{30}$ The étalon fringe effect can be minimized by various optical or electronic means. ${ }^{31,32}$ An additional low-frequency modulation (two-tone $\mathrm{FM}^{33}$ ) can be used to reduce drifts and interferences of the demodulated (from the high-frequency FM) baseline.

When the modulation index $\beta$ is of the order of unity or less, the FM spectrum can be approximated by a carrier and two first-order sidebands. If only the carrier is tuned to interact with a narrow sub-Doppler resonance, then the detection process is intrinsically dispersion sen- 
sitive. When converted to the equivalent absorption signal, the shot-noise-limited FM sensitivity becomes

$$
(\alpha L)_{\min }=\left(\frac{2 e B}{\eta P_{0}}\right)^{1 / 2} \frac{\sqrt{2}}{J_{0}(\beta) J_{1}(\beta)},
$$

where $J_{0}\left(J_{1}\right)$ is the zero (first) -order Bessel function. The modulation-dependent function $J_{0}(\beta) J_{1}(\beta)$ has its maximum value of 0.34 at $\beta \approx 1.1$. Compared with Eq. (1) for the ideal case of homodyne detection, FM heterodyne detection suffers a factor of $\sim 4$ loss in sensitivity for a fixed total optical power. This is due in part to the power reduction implied in converting some of the main carrier to sidebands and in part to the downconversion of shot noise from an additional spectral window by the second sideband. Carefully designed FM detection can often nearly reach the sensitivity limit set in Eq. (2).

The sensitive nature of FM spectroscopy along with its high resolution and high-speed detection capabilities have rendered this technique very popular. It has been used widely in nonlinear laser spectroscopy, ${ }^{34}$ including optical heterodyne saturation spectroscopy, ${ }^{27}$ two-photon FM and modulation transfer spectroscopy, ${ }^{35,36}$ Raman spectroscopy, ${ }^{37}$ and heterodyne four-wave mixing. ${ }^{38,39}$ Recent developments with tunable diode lasers have made the FM technique simpler and more accessible. The field of FM-based laser-diode detection of trace gas and remote sensing is growing rapidly.

\section{J. Noise-Immune Cavity-Enhanced Optical Heterodyne Molecular Spectroscopy}

We have seen that a shot-noise-limited absorption sensitivity of $10^{-12}-10^{-13}$ is in principle available by use of the external-cavity-enhancement technique. FM spectroscopy becomes a natural candidate to achieve such a lofty goal. In our recent development of noise-immune cavityenhanced optical heterodyne molecular spectroscopy (NICE-OHMS) we have done exactly that, by combining the FM technique with the external-cavity-enhancement approach. In the best case, when the cavityenhancement effect applies only to the signal without causing any extra noise, the sensitivity of FM spectroscopy will be improved simply by the cavity-enhancement factor, namely, $(2 \times$ finesse $/ \pi)$. Therefore we can rewrite Eq. (2) for the minimum detectable absorption as

$$
(\alpha L)_{\min }=\frac{\pi}{2 \times \text { finesse }}\left(\frac{2 e B}{\eta P_{0}}\right)^{1 / 2} \frac{\sqrt{2}}{J_{0}(\beta) J_{1}(\beta)} .
$$

A numerical example is given here to better serve the illustration. Suppose the modulation index $\beta=0.5$ and the photodiode responsivity $\eta=0.85 \mathrm{~A} / \mathrm{W}$. Also assume the total optical power $P_{0}=5 \mathrm{~mW}$ and the detection bandwidth $=1 / 2 \pi \mathrm{Hz}$, corresponding to a 1 -s time constant. Then for a plain cell the noise-equivalent integrated absorption is $(\alpha L)_{\min }=2.2 \times 10^{-8}$. Under the same conditions a cavity with a finesse of $100000 \mathrm{im}$ proves the sensitivity to $(\alpha L)_{\min }=3.5 \times 10^{-13}$. We have indeed achieved a noise-equivalent sensitivity of $5.2 \times 10^{-13}$ of an integrated absorption at 1-s averaging, corresponding to an absorption of $1 \times 10^{-14} / \mathrm{cm}$. Such a sensitivity is 4 orders of magnitude higher than any other previously reported results, according to our knowledge.

\section{NICE-OHMS PRINCIPLE}

In order to use the FM detection technique for the absorption sample located inside a high-finesse cavity we have to overcome two limitations. First, the linewidth of a highfinesse cavity is narrow. Thus the cavity is a sharp discriminator against laser-frequency noise. Any residual frequency-locking noise between the laser and the cavity is efficiently converted to amplitude noise. This leads to a serious degradation of the achievable S/N. Second, the FM sideband frequency is limited by the cavity bandwidth. Too high a modulation frequency simply reduces the coupling of the sidebands into the cavity and thereby denies their useful interactions with molecules. For a cavity with a reasonable length, say $50 \mathrm{~cm}$, and a finesse of 10000 , its linewidth (FWHM) is only $30 \mathrm{kHz}$. A modulation frequency this low seldom yields a shot-noiselimited performance. What is needed is a way to have the FM sidebands at a high frequency to get lowamplitude noise, while the cavity accepts the sidebands in exactly the same manner as it accepts the carrier so as to reduce the FM-to-AM noise conversion. In our new scheme of modulation and detection we frequency modulate the input laser beam at exactly the splitting frequency of the cavity free spectral range (FSR). We then detect and demodulate the cavity-transmitted light at the modulation frequency. The small residual frequency variations of the laser will still lead to some amplitude fluctuations and small optical phase shifts of the transmitted carrier, but they will also lead to exactly the same amplitude fluctuations and phase shifts of the sidebands that are transmitted on adjacent or nearby cavity axial orders. So the transmitted light still accurately represents an FM spectral triplet, with minimal AM conversion that is due to the relative laser/cavity frequency jitter. Thus the noise level can approach the intrinsic AM noise level of the laser at the FSR frequency, typically a few hundred megahertz or a few gigahertz.

We can refer to this property as (laser-frequency) noiseimmune detection. This modulation and detection scheme enables profitable use of the high cavity finesse without a noise penalty. Figure 1 shows the case where the central component is used to detect the intracavity molecular resonance. Initially all the FM components are lined up with their respective cavity modes. The central cavity mode will then be frequency pulled because of the change of intracavity refractive index by the molecular dispersion. The detector viewing the transmitted

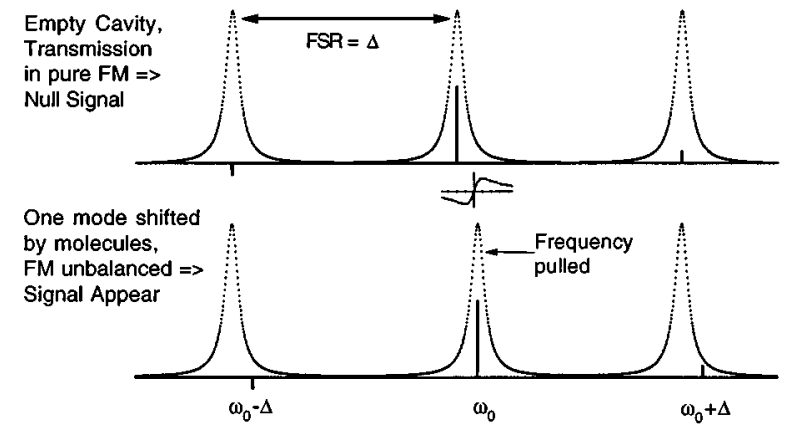

Fig. 1. Optical spectrum and the detection principle for NICEOHMS. 


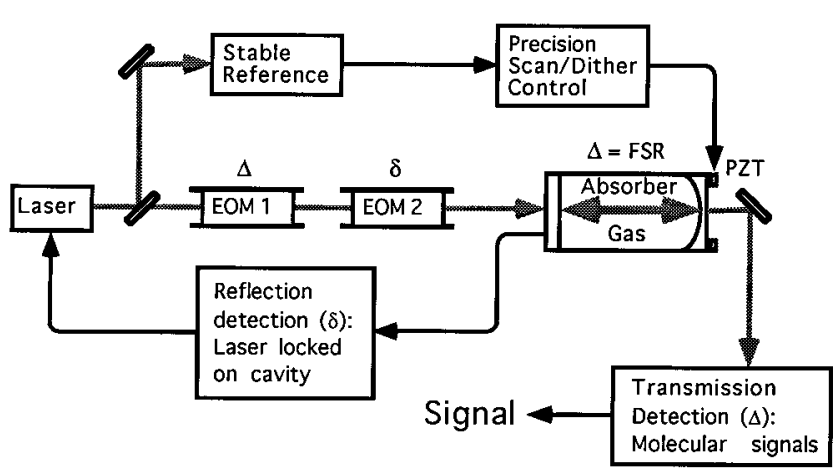

Fig. 2. General experimental schematic for the NICE-OHMS spectrometer.

light will thus generate a dispersion signal in the rf beat after the phase-sensitive demodulation.

Figure 2 shows the general experimental schematic. One may use two electro-optic phase modulators to impose two sets of FM sidebands on the laser beam. The modulation at a low-frequency $\delta$ is detected in the cavityreflection signal to produce the cavity-dispersion locking error for stabilizing the laser onto the cavity. The sidebands at the high-modulation frequency $\Delta=$ FSR are used to probe the intracavity molecular resonances and are detected in cavity transmission, after some adequate optical isolation between the cavity and the photodiode. To study the resonance signal line shape and width, a precision scanning capability for the spectrometer is important. A frequency offset locking loop is implemented to permit a sweep of the laser frequency relative to a stable reference with an $\mathrm{rf}$ resolution. During the scan the cavity FSR will change slightly. To maintain the noise-immune property, we actively track the sideband frequency to the cavity FSR value.

\section{EXPERIMENTAL RESULTS}

First we show the noise-immune detection of the $\mathrm{C}_{2} \mathrm{HD}\left(\nu_{2}+3 \nu_{3}\right) P(5)$ transition at $1.064 \mu \mathrm{m} .{ }^{40}$ To have a clear demonstration of the noise-immune nature of the detection, we deliberately set the laser/cavity lock to be loose and even oscillating; then we compare the recovered $\mathrm{S} / \mathrm{N}$ before and after the lock was sabotaged. The result is shown in Fig. 3. The cavity length was dithered at a low audio frequency, and a lock-in was used to demodulate signals from the direct cavity transmission (DC) and from the high-frequency channel of the NICE-OHMS. Signal line shapes follow modulation-broadened derivatives of absorption (DC) ${ }^{24}$ and dispersion (NICEOHMS). ${ }^{41}$ The DC detection of the intracavity molecular absorption (upper row) is shown to be critically dependent on the performance of the laser/cavity lock. (A fast laser/ cavity frequency-lock servo was used for the graphs obtained in the left column, while a slow and noisy servo was used for those in the right column.) However, increased laser-frequency noise (relative to the cavity) yields little effect in our FM detection (bottom row).

Figure 4 shows the experimental sensitivity we achieved using 1.8-mTorr $\mathrm{C}_{2} \mathrm{HD}$ gas. The cavity finesse is 100000 and the intracavity buildup power is $\sim 300 \mathrm{~W}$, giving a saturation parameter of $\sim 1.75$ and a saturation peak contrast of $13.2 \%$. The single-pass (46.9-cm-long cavity) linear absorption is $\sim 3 \times 10^{-8}$. Therefore the absolute level of saturated absorption by the intracavity molecules is $4 \times 10^{-9}$. This is verified by the DC detection of the cavity transmission, shown in the top graph of the figure. The calibration process involves measurement of cavity finesse, on-resonance transmission, and reflection dip contrast, from which we calculate the residual round-trip cavity losses. With the laser locked tightly onto the cavity with a relative linewidth of $\sim 1 \mathrm{mHz}$, the simple cavity-dither and lock-in detection of the transmission yields a S/N (amplitude/rms noise) of 130 at 1 -s averaging. This corresponds to a detection sensitivity of 3 $\times 10^{-11}$ at $1 \mathrm{~s}$. The corresponding S/N from the NICEOHMS detection is 7700 with a 1 -s time constant, as shown in the bottom graph of the figure. This translates into a noise-equivalent detection sensitivity of 5.2 $\times 10^{-13}$ at 1 -s averaging, $\sim 1.5$ times worse than the shot-noise limit calculated previously. The NICE-OHMS

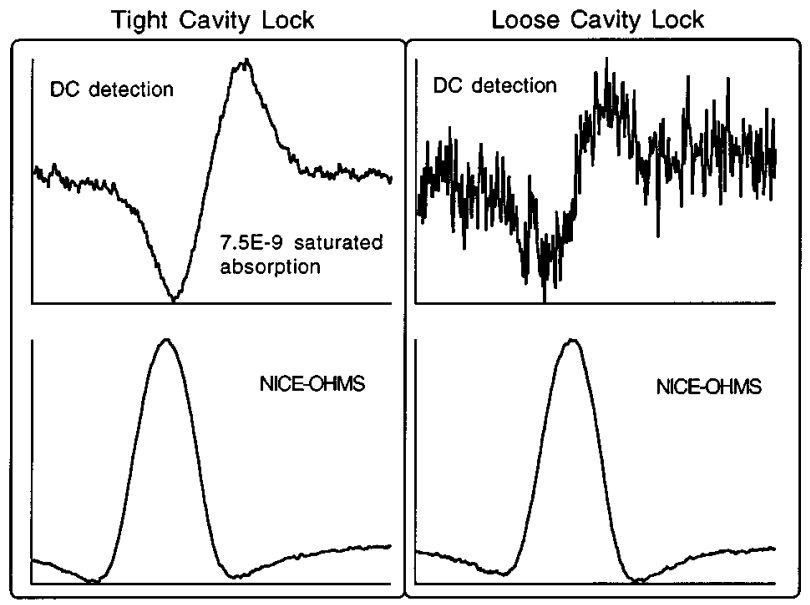

Fig. 3. Demonstration of the noise-immune property of NICEOHMS. The $\mathrm{C}_{2} \mathrm{HD}\left(\nu_{2}+3 \nu_{3}\right) P(5)$ resonance signal is recovered by both cavity-dither lock-in (DC) detection and the NICEOHMS technique, under the experimental conditions of a tight laser/cavity lock (left column) and a substantially deteriorated lock (right column).

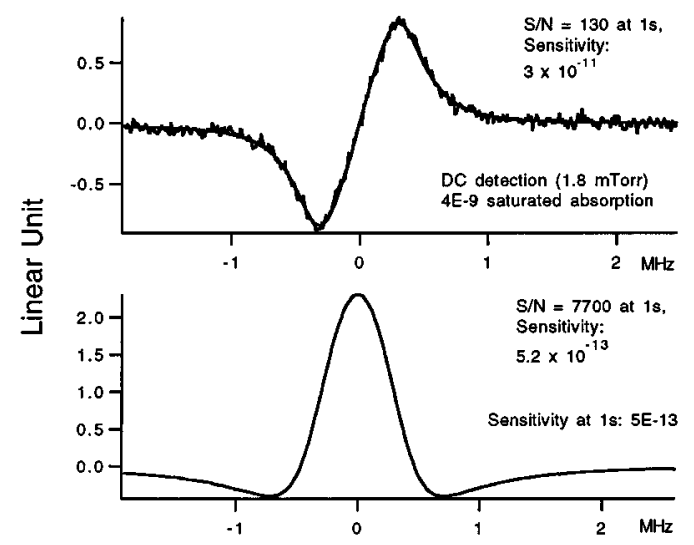

Fig. 4. Sensitivity measurement of the NICE-OHMS technique. The upper graph shows the level of the saturated absorption, while the lower graph shows the corresponding $\mathrm{S} / \mathrm{N}$ obtained via NICE-OHMS. The noise-equivalent detection sensitivities (normalized to 1 -s time constant) are $3 \times 10^{-11}$ for cavity-dither detection and $5.2 \times 10^{-13}$ for NICE-OHMS. 
result is $\sim 60$ times better than the straightforward dither detection, basically because of its higher modulation frequency (319-MHz FM sideband frequency compared with $500-\mathrm{Hz}$ dither frequency) and its insensitivity toward the laser-frequency noise relative to the cavity.

We have to point out that although the requirement of the laser/cavity locking is much more relaxed for NICEOHMS than in the simple DC detection, the laser linewidth still needs to be narrowed so that a stable optical power is effectively coupled into the cavity. For a cavity linewidth of, say, $10 \mathrm{kHz}$, it should be straightforward to lock a commercially available external-cavity diode laser below $100 \mathrm{~Hz}$ relative to the cavity, using a simple feedback control of laser-diode current and its external grating. Another important technical issue is that the recovered line shape is influenced by a residual AM associated with FM at the cavity FSR frequency. With an active control loop we are able to eliminate the residual amplitude modulation ${ }^{42}$ and obtain a line shape matching perfectly to a theoretical model, resulting in a flat and nearly shot-noise-limited background for the fit residual throughout the entire tuning range of the resonance.

With this ultrasensitive capability of our spectrometer we naturally seek other weak transitions within the tuning range of the $\mathrm{Nd}$ :YAG laser at $1.064 \mu \mathrm{m}$. We have measured two such lines, namely, ${ }^{12} \mathrm{C}_{2} \mathrm{H}_{2}\left(2 \nu_{1}+\nu_{2}\right.$ $\left.+\nu_{5}\right) R(12)^{43}$ and ${ }^{12} \mathrm{C}^{16} \mathrm{O}_{2}\left(2 \nu_{1}+3 \nu_{3}\right) R(6),{ }^{44}$ with their respective transition dipole moment of $50 \mu \mathrm{D}$ and $6 \mu \mathrm{D}$ $\left(1 \mathrm{D}=3.33564 \times 10^{-30} \mathrm{C} \cdot \mathrm{m}\right)$. They are both weaker than the $\mathrm{C}_{2} \mathrm{HD}\left(\nu_{2}+3 \nu_{3}\right) P(5)$ transition, which has a transition dipole moment of $\sim 70 \mu \mathrm{D}$.

Using the same gas pressure, optical power, and cavitydither amplitude, we compare the saturated absorption signals of $\mathrm{CO}_{2}, \mathrm{C}_{2} \mathrm{HD}$, and $\mathrm{C}_{2} \mathrm{H}_{2}$ in Fig. 5. The $\mathrm{C}_{2} \mathrm{H}_{2}$ transition is recovered with an excellent $\mathrm{S} / \mathrm{N}$, as shown in Fig. 5(c). The signal size is roughly $1 / 4.3$ of that for $\mathrm{C}_{2} \mathrm{HD}$, using the same gas pressure and optical power. It provides another frequency reference for the Nd:YAG laser. The center frequency of the resonance is $281612403.278(0.025) \mathrm{MHz}$, i.e., it is $17708.458(0.014)$ $\mathrm{MHz}$ red of a frequency-doubled Nd:YAG laser locked on the $a_{10}$ hyperfine-structure component of the $R(56) 32-0$ $\mathrm{I}_{2}$ transition. ${ }^{45}$ The pressure broadening rate of $34(1)$ $\mathrm{MHz} /$ Torr is similar to that of $\mathrm{C}_{2} \mathrm{HD} .{ }^{40}$

For the $\mathrm{CO}_{2}$ transition, however, the saturated absorption signal is much weaker than the $\mathrm{C}_{2} \mathrm{HD}$ line, by more than a factor of 350. This $\mathrm{CO}_{2}$ resonance involves two quanta of symmetric stretch and three quanta of antisymmetric stretch of the $\mathrm{C}-\mathrm{O}$ bond. ${ }^{44}$ The bending mode $\left(\nu_{2}\right)$ is not excited. The lifetime of the excited vibrational state is estimated to be $\sim 2 \mathrm{~ms}$, mainly because of the deexcitation via the vibrational transition $\left(2,0^{\circ}, 3\right) \rightarrow\left(2,0^{\circ}, 2\right)$. The relevant molecular constants are $\left(\mathrm{cm}^{-1}\right) \quad B^{\prime}=0.39021894, \quad D^{\prime}=1.33373$ $\times 10^{-7}$ for the ground state, and $B^{\prime \prime}=0.38234, D^{\prime \prime}$ $=1.71$ for the excited state with the band origin at 9388.990. ${ }^{46}$

Under exactly the same experimental conditions (except for the change of sample gas), the recovered line shape associated with the ${ }^{12} \mathrm{C}^{16} \mathrm{O}_{2}\left(2 \nu_{1}+3 \nu_{3}\right) R(6)$ transition is vastly different from either $\mathrm{C}_{2} \mathrm{HD}$ or $\mathrm{C}_{2} \mathrm{H}_{2}$, as indicated in Fig. 5. In fact, our theoretical model for the nearly perfect fit of the experimental data involves two separate resonances that have different line centers and line widths. More importantly, the narrower negativegoing peak indicates a physical process reversed from the normal saturated absorption. The intracavity optical power is $410 \mathrm{~W}$, where the saturation of this $\mathrm{CO}_{2}$ transition is estimated to be only a few percent $(2 \%)$ in the freeflight regime. As we reduce the input power by a factor of 4 , the resonance line shape remains relatively unchanged. The signal size, however, reduces roughly by the square of the power change. The line shape does not depend on the laser-dither frequency either. The change of the dither amplitude, on the other hand, will surely have a larger impact on the signal size of the relatively wider peak, thereby causing the apparent change on the signal line shape. This change is well accounted for by our theoretical model, and our fit is able to produce nearly consistent linewidths corresponding to a certain gas pressure.

As we change the intracavity gas pressure, we witness linewidth broadenings and line-center shifts for both peaks. Extrapolated to zero pressure, the inverted peak has a FWHM of $\sim 100 \mathrm{kHz}$, while the normal peak has a FWHM of $410 \mathrm{kHz}$. The width of the normal peak is almost twice the transit linewidth, which would usually be obtained in the absence of the interaction between the two resonances. The two line centers are pushed apart as the pressure increases. The zero-crossing point shows relatively small shifts caused by pressure. These results are shown in Fig. 6.

(a)
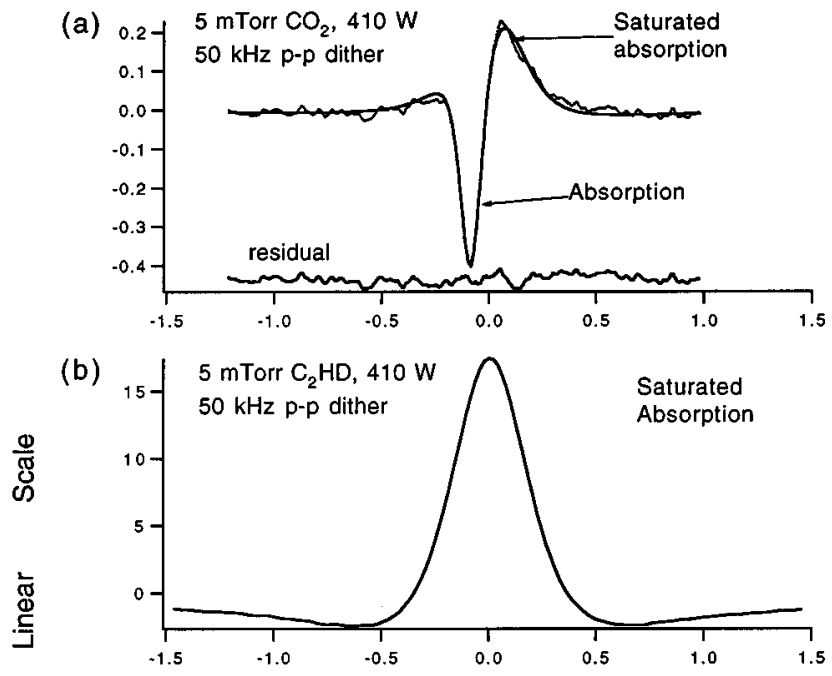

(c)

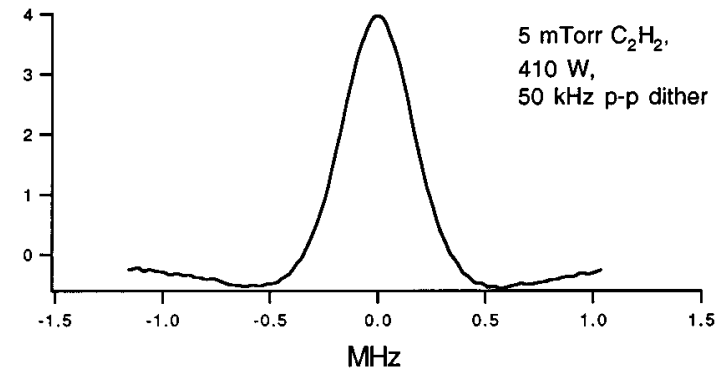

Fig. 5. Line-shape comparison among the resonances of (a) $\mathrm{CO}_{2}$, (b) $\mathrm{C}_{2} \mathrm{HD}$, and (c) $\mathrm{C}_{2} \mathrm{H}_{2}$, under the same experimental conditions. 


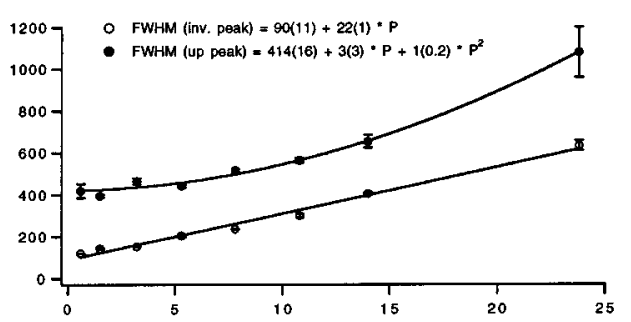

Fig. 6. Pressure-dependent linewidth changes for both $\mathrm{CO}_{2}$ resonances. The negative-going peak has a zero-pressure extrapolated linewidth of $\sim 100 \mathrm{kHz}$, half the value of the transit time broadening. These aspects lead us to tentatively identify this feature as arising from two-photon absorption.

Since our detection scheme involves a pair of FM sidebands lying one cavity FSR away from the laser carrier, it is natural to suspect that maybe the asymmetrical line shape is caused by some coincidental resonances located just at the sideband frequencies or at the crossovers between the carrier and one of the sidebands. We tuned the laser frequency around those places and found no such lucky resonances. One likely explanation for the abnormal line shape is the existence of a near-resonant two-photon transition in the neighborhood of the onephoton resonance. This is supported by the fact that the zero-pressure linewidth of the inverted peak is only $\sim 100 \mathrm{kHz}$, half of that dictated by the transit time broadening $(210 \mathrm{kHz})$. It is as if, during the transit, the molecule experiences twice as many radians of signal phase, for example, by resonating with a two-photon response. A second possible physical effect is the existence of some quantum interference caused by the state mixing of the excited vibrational state.

\section{SUMMARY, DISCUSSIONS, AND OUTLOOK}

A record high detection sensitivity has been achieved by the NICE-OHMS technique in the work on molecular overtone transitions at $1.064 \mu \mathrm{m}$ with a cavity finesse of 100000 . With a single-pass integrated (saturated) absorption at the level of $4 \times 10^{-9}$, the recovered $\mathrm{S} / \mathrm{N}$ is $\sim 7700$, normalized to 1 -s averaging time. This corresponds to a noise-equivalent absorption detection sensitivity of $5.2 \times 10^{-13}\left(1 \times 10^{-14} / \mathrm{cm}\right)$ at $1 \mathrm{~s}$. Stated equivalently, the detection sensitivity for the refractive index is of the order of $1 \times 10^{-19}$ at $1 \mathrm{~s}$.

It is amusing to illustrate this kind of detection sensitivity with a more conventional model of two-level atoms. A two-level atom has an absorption cross section of $\hbar^{2} \approx 10^{-10} \mathrm{~cm}^{2}$ for its resonance line. If we assume the spatial density of the atoms is $\sim 1$ atom $/ \mathrm{cm}^{3}$, then in a 50 -cm absorption length (about the length of our cavity) the integrated absorption will be $5 \times 10^{-9}$. It is at this level of absorption that we are getting a $\mathrm{S} / \mathrm{N}$ of 9000 at 1 s. The density of 1 atom $/ \mathrm{cm}^{3}$ corresponds to a pressure of $3 \times 10^{-17}$ Torr $\left(4 \times 10^{-15} \mathrm{~Pa}\right)$. The equivalent number of atoms inside the mode volume of our intracavity light field is $\sim 0.13$. The minimum detectable absorption signal will thus correspond to an atom density of $1 / 9000 \mathrm{~cm}^{-3}$, or a pressure of $3.4 \times 10^{-21}$ Torr (4.5 $\times 10^{-19} \mathrm{~Pa}$ ). This result is of course not realistic, owing to the saturation of the relatively strong transitions associated with two-level atoms, demanding a low input power to the high- $Q$ cavity. If we reduce the input power by a factor of $10^{8}$ to avoid saturation, then the sensitivity as expressed in Eq. (3) will correspondingly decrease by $10^{4}$, and we will be able to detect just a single atom in a $1-\mathrm{cm}^{3}$ volume.

To further enhance the detection sensitivity, a direct approach is of course to build a cavity with an even higher finesse. With the present cavity having a finesse of 100000 the potential for growth along this direction is limited. Finding a fast photodetector that is able to handle a large optical power, say, $10 \mathrm{~mW}$, will also increase the detection $\mathrm{S} / \mathrm{N}$. However, in the heterodyne detection, the shot-noise level is set mainly by the local oscillator, i.e., the leakage power from the cavity, which has prepared the molecular signal, but is not a molecular signal itself. In the ideal case, we should have the signal itself set the shot-noise level. All other power, after preparing the molecules, should be avoided in detection. In this way the shot-noise floor will be extremely small. From this point of view it seems to be advantageous to detect the molecular signal in cavity reflection, provided that the optical impedance match of the cavity is adequate. The power of the local oscillator is reduced by a large factor because of the destructive interference between the cavity-leaked field and the direct reflection of the incident field. Assuming that similar proportions of the molecular signal travel through either exit of the cavity, with a much reduced shot-noise floor we can thus expect an enhanced detection $\mathrm{S} / \mathrm{N}$. Our preliminary result of the reflection detection actually produced a less favorable $\mathrm{S} / \mathrm{N}$, limited by noise of technical origins. In the transmission detection the spatial mode cleaning and matching of the local oscillator with respect to the molecular signal is achieved automatically by the cavity itself. This ideal condition, however, is compromised to a certain degree in the case of reflection detection, owing to several causes. The incident field has a certain level of pointing instability that is due to the laser source. The original nearly ideal Gaussian mode structure of the field could also be slightly damaged along the optical path. This mode spatial variance, often time dependent, leads directly to the amplitude noise in the subsequent photodiode detection and $\mathrm{rf}$ demodulation. A reasonable approach to solve this problem would be to use an auxiliary optical cavity placed just before the high-finesse cavity to stabilize the mode direction and the structure of the incident field.

At present the instrumental resolution bandwidth of our spectrometer still exceeds the natural linewidth of the molecular resonance. Although such a transit-timelimited linewidth can be narrowed by optical selection of slow-moving molecules, the result comes with a penalty in the reduction of $\mathrm{S} / \mathrm{N}$. To have a longer interaction time, we either need heavier molecules or to use an optical cavity with a larger mode size. Construction of a highfinesse cavity with a large mode size could be technically challenging. For our 1.064- $\mu \mathrm{m}$ standing-wave cavity we will soon try to replace the mirror of 1-m radius of curvature with a 50-m radius one. The other mirror is flat. The cavity finesse will remain the same, but the mode 
size should increase threefold, producing a transit limit of $\sim 95 \mathrm{kHz}$ for $\mathrm{C}_{2} \mathrm{HD}$ molecules. More exotic multiplemirror cavity designs involving astigmatism-compensated intracavity beam expansion are also under consideration. A more straightforward and less expensive approach could be by use of a pressure-tuned high-finesse mirror to achieve the desired radius for operation. ${ }^{47}$

A high detection sensitivity along with a high resolution brings us precious opportunities to explore new phenomena that are otherwise hidden from us. An ongoing collaboration between JILA and MIT (R. W. Field's group) is to utilize the available sensitivity and resolution of our spectrometer to search for the predicted permutation splitting associated with exchange of the hydrogen atoms when their vibrational amplitudes become significantly large in the high-order overtone band $\left(5 \nu_{3}\right)$ of ${ }^{13} \mathrm{C}_{2} \mathrm{H}_{2} \cdot{ }^{48}$ Another interesting project is the test of the symmetrization postulate of quantum mechanics. Using diode-laser absorption spectroscopy with ${ }^{16} \mathrm{O}_{2}$ molecules, scientists have demonstrated that if there is any violation of BoseEinstein statistics by the identical spin-0 oxygen nuclei, it does not exceed a part in a million, the sensitivity of their spectrometer. ${ }^{49}$ The possible violation of the Bose statistics can of course be tested at a sensitivity one million times higher than these reported results.

The NICE-OHMS detection technique can also be applied to the case of two-photon resonances. When the optical intensity is reduced, the fluorescence $\mathrm{S} / \mathrm{N}$ decreases as $I^{-1}$ while the NICE-OHMS S/N decreases as $I^{-1 / 2}$. Therefore NICE-OHMS should win at the low-intensity range, while the fluorescence detection may be advantageous at a high power. Using a relatively low optical power is advantageous, in the point of view for a frequency standard, as the power-related systematic shifts on the center frequency can be reduced. Ordinarily the direct absorption, i.e., the change in transmitted light that is due to such a two-photon transition, would be small and difficult to detect. However, with the high sensitivity available from the NICE-OHMS, it compares favorably when a low light level is used for detection, and it is especially valuable in the case when the fluorescence is weak and the fluorescence-detection efficiency (quantum efficiency and/or collection efficiency) is low.

In the work of optical frequency metrology, the NICEOHMS technique can provide us with thousands of weak molecular lines as high-quality visible-frequency/ wavelength references. We will soon start to construct an optical frequency network covering the important spectral region around $790 \mathrm{~nm}$ using overtone transitions of $\mathrm{C}_{2} \mathrm{H}_{2}, \mathrm{C}_{2} \mathrm{HD}$, and $\mathrm{NH}_{3} .{ }^{50}$

\section{ACKNOWLEDGMENTS}

We wish to acknowledge the funding support from the National Institute of Standards and Technology, the U.S. Office of Naval Research, the U.S. Air Force Office of Scientific Research, and the National Science Foundation.

\footnotetext{
*Present address: Quantum Optics Group, Caltech, Pasadena, California.

${ }^{\dagger}$ Present address: Department of Physics, East China Normal University, Shanghai, China.
}

${ }^{\ddagger}$ Staff member, Quantum Physics Division, National Institute of Standards and Technology.

\section{REFERENCES}

1. J. L. Hall, L. Hollberg, L.-S. Ma, T. Baer, and H. G. Robinson, "Progress toward phase-stable optical frequency standards," J. Phys. (France) Colloq. 42, Suppl. 12, C8 59-71 (1981).

2. See, for example, Ultrasensitive Laser Spectroscopy, D. S. Kliger, ed. (Academic, New York, 1983).

3. A. C. Tam, "Photothermal spectroscopy as a sensitive spectroscopic tool," in Optical Methods for Ultrasensitive Detection and Analysis: Techniques and Applications, B. L. Fearey, ed., Proc. SPIE 1435, 114-127 (1991).

4. C. E. Wieman and T. W. Hänsch, "Doppler-free laser polarization spectroscopy," Phys. Rev. Lett. 36, 1170-1173 (1976); M. D. Levenson and G. L. Eesley, "Polarization selective optical heterodyne detection for dramatically improved sensitivity in laser spectroscopy," Appl. Phys. 19, 1-17 (1979).

5. W. M. Fairbank, Jr., T. W. Hänsch, and A. L. Schawlow, "Absolute measurement of very low sodium-vapor densities using laser resonance fluorescence," J. Opt. Soc. Am. 65, 199-204 (1975).

6. W. Neuhauser, M. Hohenstatt, P. Toscheck, and H. Dehmelt, "Optical sideband cooling of visible atom cloud confined in parabolic well," Phys. Rev. Lett. 41, 233-236 (1978).

7. D. J. Wineland, W. M. Itano, J. J. Bollinger, J. C. Bergquist, and H. Hemmati, "Spectroscopy of stored ions using fluorescence techniques," in Laser-Based Ultrasensitive Spectroscopy and Detection V, R. A. Keller, ed., Proc. SPIE 426, 65-70 (1983).

8. E. B. Shera, N. K. Seitzinger, L. M. Davis, R. A. Keller, and S. A. Soper, "Detection of single fluorescent molecules," Chem. Phys. Lett. 174, 553-557 (1990).

9. B. A. Bushaw, T. J. Whitaker, B. D. Cannon, and R. A. Warner, "Time-interval distribution and photon-burstcorrelation spectroscopy of a strontium atomic beam," J. Opt. Soc. Am. B 2, 1547-1553 (1985).

10. G. S. Hurst, M. G. Payne, S. D. Kramer, and J. P. Young, "Resonance ionization spectroscopy and one-atom detection," Rev. Mod. Phys. 51, 767-819 (1979).

11. G. D. Houser and E. Garmire, "Balanced detection technique to measure small changes in transmission," Appl. Opt. 33, 1059-1062 (1994).

12. K. L. Haller and P. C. D. Hobbs, "Double beam laser absorption spectroscopy: shot noise-limited performance at baseband with a novel electronic noise canceller," in Optical Methods for Ultrasensitive Detection and Analysis: Techniques and Applications, B. L. Fearey, ed., Proc. SPIE 1435, 298-309 (1991).

13. J. Altmann, R. Baumgart, and C. Weitkamp, "Two-mirror multipass absorption cell," Appl. Opt. 20, 995-999 (1981).

14. A. Kastler, "Atomes à l'intérieur d'un interféromètre PerotFabry," Appl. Opt. 1, 17-24 (1962).

15. P. Cerez, A. Brillet, C. N. Man-Pichot, and R. Felder, "He-Ne lasers stabilized by saturated absorption in iodine at $612 \mathrm{~nm}$," IEEE Trans. Instrum. Meas. TIM-29, 352-354 (1980).

16. L.-S. Ma and J. L. Hall, "Optical heterodyne spectroscopy enhanced by an external optical cavity: toward improved working standards," IEEE J. Quantum Electron. 26, 20062012 (1990).

17. M. De Labachelerie, K. Nakagawa, and M. Ohtsu, "Ultranarrow ${ }^{13} \mathrm{C}_{2} \mathrm{H}_{2}$ saturated-absorption lines at $1.5 \mu \mathrm{m}$," Opt. Lett. 19, 840-842 (1994).

18. J. L. Hall and C. J. Bordé, "Shift and broadening of saturated absorption resonances due to curvature of the laser wave fronts," Appl. Phys. Lett. 29, 788-790 (1976).

19. A. O'Keefe and D. A. G. Deacon, "Cavity ring-down optical spectrometer for absorption measurements using pulsed laser sources," Rev. Sci. Instrum. 59, 2544-2551 (1988). 
20. D. Romanini and K. K. Lehmann, "Cavity ring-down overtone spectroscopy of $\mathrm{HCN}, \mathrm{H}^{13} \mathrm{CN}$, and $\mathrm{HC}^{15} \mathrm{~N}$," J. Chem. Phys. 102, 633-642 (1995).

21. T. W. Hänsch, A. L. Schawlow, and P. E. Toschek, "Ultrasensitive response of a cw dye laser to selective extinction," IEEE J. Quantum Electron. QE-8, 802-804 (1972).

22. H. J. Kimble, "Calculated enhancement for intracavity spectroscopy with a single-mode laser," IEEE J. Quantum Electron. QE-16, 455-461 (1980).

23. V. M. Baev and P. E. Toschek, "Sensitivity limits of laser intracavity spectroscopy," in Optical Methods in Atmospheric Chemistry, H. I. Schiff and U. Platt, eds., Proc. SPIE 1715, 381-392 (1993).

24. H. Wahlquist, "Modulation broadening of unsaturated Lorentzian lines," J. Chem. Phys. 35, 1708-1710 (1961).

25. B. Smaller, "Precise determination of the magnetic moment of the deuteron," Phys. Rev. 83, 812-820 (1951); R. V. Pound, "Electronic frequency stabilization of microwave oscillators," Rev. Sci. Instrum. 17, 490-505 (1946).

26. G. C. Bjorklund, "Frequency-modulation spectroscopy: a new method for measuring weak absorptions and dispersions," Opt. Lett. 5, 15-17 (1980).

27. J. L. Hall, L. Hollberg, T. Baer, and H. G. Robinson, "Optical heterodyne saturation spectroscopy," Appl. Phys. Lett. 39, 680-682 (1981); R. W. P. Drever, J. L. Hall, F. V. Kowalski, J. Hough, G. M. Ford, A. J. Munley, and H. Ward, "Laser phase and frequency stabilization using an optical resonator," Appl. Phys. B 31, 97-105 (1983).

28. M. Gehrtz, G. C. Bjorklund, and E. A. Whittaker, "Quantum-limited laser frequency-modulation spectroscopy," J. Opt. Soc. Am. B 2, 1510-1525 (1985).

29. P. Werle, "Laser excess noise and interferometric effects in frequency-modulated diode-laser spectrometers," Appl. Phys. B 60, 499-506 (1995).

30. N. C. Wong and J. L. Hall, "Servo control of amplitude modulation in FM spectroscopy: demonstration of shotnoise limited detection," J. Opt. Soc. Am. B 2, 1527-1533 (1985).

31. L.-G. Wang, D. A. Tate, H. Riris, and T. F. Gallagher, "High-sensitivity frequency-modulation spectroscopy with a GaAlAs diode laser," J. Opt. Soc. Am. B 6, 871-876 (1989).

32. D. R. Hjelme, S. Neegård, and E. Vartdal, "Optical interference fringe reduction in frequency-modulation spectroscopy experiments," Opt. Lett. 20, 1731-1733 (1995).

33. G. R. Janik, C. B. Carlisle, and T. F. Gallagher, "Two-tone frequency-modulation spectroscopy," J. Opt. Soc. Am. B 3, 1070-1074 (1986).

34. G. C. Bjorklund and M. D. Levenson, "Sub-Doppler frequency-modulation spectroscopy of $I_{2}$," Phys. Rev. A 24, 166-169 (1981).

35. W. Zapka, M. D. Levenson, F. M. Schellenberg, A. C. Tam, and G. C. Bjorklund, "Continuous-wave Doppler-free twophoton frequency-modulation spectroscopy in Rb vapor," Opt. Lett. 8, 27-29 (1983).

36. L. S. Ma, L. E. Ding, and Z. Y. Bi, "Doppler-free two-photon modulation transfer spectroscopy in sodium dimers," Appl. Phys. B 51, 233-237 (1990).
37. G. J. Rosasco and W. S. Hurst, "Phase-modulated stimulated Raman spectroscopy," J. Opt. Soc. Am. B 2, 14851496 (1985).

38. J. H. Shirley, "Modulation transfer process in optical heterodyne saturation spectroscopy," Opt. Lett. 7, 537-539 (1982).

39. J. Bialas, R. Blatt, W. Neuhauser, and P. E. Toschek, "Ultrasensitive detection of light absorption by few ions," Opt. Commun. 59, 27-30 (1986).

40. J. Ye, L.-S. Ma, and J. L. Hall, "Ultra-stable optical frequency reference at $1.064 \mu \mathrm{m}$ using a $\mathrm{C}_{2} \mathrm{HD}$ molecular overtone transition," IEEE Trans. Instrum. Meas. 46, 178 (1997); J. Ye, "Ultrasensitive high resolution laser spectroscopy and its application to optical frequency standards," Ph.D. dissertation (University of Colorado at Boulder, Boulder, Colorado, 1997).

41. R. L. Smith, "Practical solutions of the lock-in detection problem for Lorentz and dispersion resonance signals," J. Opt. Soc. Am. 61, 1015-1022 (1971).

42. J. L. Hall, J. Ye, L.-S. Ma, K. Vogel, and T. Dinneen, "Optical frequency standards: progress and applications," in Laser Spectroscopy XIII, Y. Z. Wang, ed. (World Scientific, Singapore, 1997).

43. K. Nakagawa, T. Katsuda, A. S. Shelkovnikov, M. de Labachelerie, and M. Ohtsu, "Highly sensitive detection of molecular absorption using a high finesse optical cavity," Opt. Commun. 107, 369-372 (1994).

44. P. Fritschel and R. Weiss, "Frequency match of the Nd:YAG laser at $1.064 \mu \mathrm{m}$ with a line in $\mathrm{CO}_{2}$," Appl. Opt. 31, 19101912 (1992).

45. M. L. Eickhoff and J. L. Hall, "Optical frequency standard at $532 \mathrm{~nm}$," IEEE Trans. Instrum. Meas. 44, 155-158 (1995); P. Jungner, M. Eickhoff, S. Swartz, J. Ye, J. L. Hall, and S. Waltman, "Absolute frequency of the molecular iodine transition $R(56) 32-0$ near 532 nm," IEEE Trans. Instrum. Meas. 44, 151 (1995).

46. L. S. Rothman and L. D. G. Young, "Infrared energy levels and intensities of carbon dioxide. II," J. Quantum Spectrosc. Radiat. Transf. 25, 505-524 (1981).

47. U. J. Greiner and H. H. Klingerberg, "Thermal lens correction of a diode-pumped Nd:YAG laser of high $\mathrm{TEM}_{00}$ power by an adjustable-curvature mirror," Opt. Lett. 19, 12071209 (1994).

48. M. Jacobson and R. W. Field, MIT, Cambridge, Mass. 02139 (private communications, 1996).

49. M. de Angelis, G. Gagliardi, L. Gianfrani, and G. M. Tino, "Test of the symmetrization postulate for spin-0 particles," Phys. Rev. Lett. 76, 2840-2843 (1996); R. C. Hilborn and C. L. Yuca, "Spectroscopic test of the symmetrization postulate for spin-0 nuclei," Phys. Rev. Lett. 76, 2844-2847 (1996).

50. L.-S. Ma, J. Ye, P. Dubé, and J. L. Hall, "Ultrasensitive FM spectroscopy enhanced by a high finesse optical cavity: application to overtone transitions of $\mathrm{C}_{2} \mathrm{H}_{2} \& \mathrm{C}_{2} \mathrm{HD}$, ” J. Opt. Soc. Am. B (to be published). 